\title{
How to see an antistar
}

\author{
A.D. Dolgov, ${ }^{1,2,3,4}$ V. A. Novikov, ${ }^{2,1,5,6}$ and M. I. Vysotsky ${ }^{2,1,5,6}$ \\ ${ }^{1}$ Novosibirsk State University, Novosibirsk, 630090, Russia \\ 2 A.I. Alikhanov Institute of Theoretical and Experimental Physics, Moscow, 113259, Russia \\ ${ }^{3}$ Dipartimento di Fisica e Scienze della Terra, Università degli Studi di Ferrara \\ Polo Scientifico e Tecnologico - Edificio C, Via Saragat 1, 44122 Ferrara, Italy \\ ${ }^{4}$ Istituto Nazionale di Fisica Nucleare (INFN), Sezione di Ferrara \\ Polo Scientifico e Tecnologico - Edificio C, Via Saragat 1, 44122 Ferrara, Italy \\ 5 Moscow Engineering Physics Institute, 115409, Moscow, Russia \\ ${ }^{6}$ Moscow Institute of Physics and Technology, 141700, Dolgoprudny, Moscow Region, Russia
}

\begin{abstract}
Polarization of photons emitted in weak decays occuring at distant star allows to determine whether this star is made from antimatter. Even more promissing is the observation of neutrinos (antineutrinos) produced at neutronization (antineutronization) reactions at the beginning of SN $(\overline{S N})$ explosion.
\end{abstract}

According to the Standard Cosmological Model (SCM) no primordial antimatter remains in the Universe. Let us shortly remind the arguments which lead to this conclusion. When in the course of the post Big Bang expansion the universe cooled down below the QCD phase transition at $T_{Q C D}=100-200 \mathrm{MeV}$, baryon-antibaryon pairs started to annihilate. If the baryon number of the Universe at these temperatures was locally zero, then the remaining frozen concentration of baryons would be (see e.g. ref. [1]):

$$
n_{B} / n_{\gamma} \approx 10^{-20}
$$

where $n_{B}$ is the number density of baryons, by assumption equal to that of antibaryons, and $n_{\gamma}$ is the number density of photons in CMB. This result is by factor $10^{11}$ smaller than the presently observed baryon concentration, which can be e.g. deduced from the recent Planck data [2], as:

$$
\eta=n_{B} / n_{\gamma} \approx 6 \times 10^{-10},
$$

with the precision at the per cent level. Here it is implicitly assumed that the amount of antibaryons is negligibly small, $n_{\bar{B}} \ll n_{B}$.

In order to avoid conclusion (1) we have either to assume that at the era of baryon-antibaryon annihilation the universe was predominantly and homogeneously populated by baryons, or that the universe has domain structure with spatially separated domains of matter and antimatter. In the last case it might be even not excluded that the total baryonic number of the universe is zero.

In the frameworks of the SCM the first option is accepted, which has a strong support from the baryogenesis theory, whose basic principles have been formulated by Sakharov almost half a century ago [3]. In all known scenarios of baryogenesis an excess of baryons over antibaryons was generated at very (or rather) high temperatures, while at the subsequent cosmological expansion and cooling down the baryon-to-photon ratio (2) stayed approximately constant, up to the entropy release by the massive particle annihilation.

In the universe with an excess of baryons a chance for antibaryons to survive was negligibly small, though in the early universe there were almost equal number densities of baryons and antibaryons, $\left(n_{B}-n_{\bar{B}}\right) / n_{B} \approx \eta \ll 1$. The temperature at which the "massacre" of antibaryons by dominant baryons stopped is fixed by the annihilation freezing which is determined by the time when the annihilation rate became equal to the cosmological expansion rate:

$$
\frac{1}{\sigma v \eta T_{f}^{3}}=\frac{M_{p}}{m_{p} T_{f}}, T_{f}=m_{p} \sqrt{\frac{m_{p}}{100 M_{p} \eta}} \approx 1 \mathrm{keV}
$$

where $\sigma v \approx 1 / m_{\pi}^{2}$ is the cross-section of $p \bar{p} \rightarrow n \pi$ reaction times the proton velocity in c.m. system, $m_{p}$ is the proton mass, $M_{p}$ is the Planck mass and $n$ is the number of pions produced in $p \bar{p}$ annihilation.

That is why the remaining antiproton concentration being proportional to

$$
\exp \left(-m_{p} / T_{f}\right) \sim 10^{-400000}
$$

is unobservably small: there is not a single primordial antiproton in all presently visible part of our Universe. This bound is evidently too strong. Statistical fluctuations of the antibaryonic density could strongly violate it but still the amount of the primordial antiprotons would remain negligible.

The fluxes of the observed in cosmic rays antiprotons and positrons are about 4 orders of magnitude smaller than the fluxes of protons and electrons respectively. They are believed to be of secondary origin, produced in catastrophic processes in stars and in interactions of energetic cosmic ray protons and electrons with matter. 
No antinuclei are observed in cosmic rays. The flux of secondary produced anti-deuterium is estimated as [4]:

$$
F_{\bar{D}} \sim 10^{-7} \mathrm{~m}^{-2} \mathrm{~s}^{-1} \mathrm{sr}^{-1}(\mathrm{GeV} / \mathrm{n})^{-1},
$$

where Gev/ $n$ is kinetic energy per nucleon. In other words, the predicted flux of $\bar{D}$ would be 5 orders of magnitude lower than the flux of antiprotons. According to the estimates of the same paper [4], the fluxes of secondary produced ${ }^{3} \bar{H} e$ and ${ }^{4} \bar{H} e$ are respectively 4 and 8 orders of magnitude smaller than the flux of $\bar{D}$. A registration of antinuclei with the flux above those predictions would be an unambiguous proof of existence of primordial cosmic antimatter. An active search of cosmic antinuclei was and is performed at several balloon (BESS) and satellite (AMS, PAMELA) missions. No single event of observation of anti-helium or any heavier antinuclei was reported. The best up-to-date limit on the anti-helium flux was reported by BESS [5] and it is: $\bar{H} e / H e<7 \times 10^{-8}$. Potentially PAMELA and AMS might improve this limit by an order and two orders of magnitude respectively. There are some new projects with even larger sensitivity. An observational limit on the flux of $\bar{D}$ was obtained in [6] by far above the theoretical expectation of the secondary production flux.

An indirect signature of cosmic antimatter could be a flux of low energy, $~(0.1-1) \mathrm{GeV}$, photons, originating from $\bar{p} p$-annihilation. There is no evidence of any excess of such radiation which demands for its explanation the annihilation source. So these data are used for quite restrictive limits on cosmologically large clumps of antimatter. In particular, the nearest anti-galaxy could be at least at the distance of 10 Mpc from us [7]. Similar considerations allow to conclude that the fraction of antimatter in colliding galaxies of Bullet cluster is smaller than $3 \times 10^{-6}$ [8]. As is was shown in ref. [9], in baryo-symmetric universe with cosmologically large domains of matter and antimatter the nearest domain of antimatter should be farther than a Gigaparsec away.

All these bounds are applicable if matter and antimatter populate the universe in the similar forms: clouds of gas and antigas, stars and antistars of the same types, etc. However, it is possible to modify [10] the baryogenesis scenario in such a way that antimatter would be mostly hidden in compact stellar type objects, which could be in our Galaxy, even in close vicinity to us. According to the suggested mechanism these objects were created in very early universe, long before the recombination, and thus the usual CMB or LSS bounds on antimatter are not directly applicable to them. These stellar-like (anti-)objects might be abundant in the universe and even make a noticeable contribution to the cosmological dark matter.

To make the paper self-contained we briefly present main features of such a model. The starting point is the Affleck-Dine (AD) mechanism of baryogenesis [11], where a scalar field $\chi$ with non-zero baryonic number has the potential with flat directions. In the course of an early cosmological evolution $\chi$ might acquire a large expectation value along the flat direction and at a later stage, when $\chi$ became massive its decay could create a large baryon asymmetry, $\eta$, which in AD-model could be even of the order of unity. To make the scenario compatible with the data one has to invent a mechanism to suppress $\eta$ down to the observed value. However, it is possible to modify the AD-mechanism in a simple way, so that a large $\eta$ was generated only in a small fraction of the total space. To realize such a picture it is sufficient to add a general renormalizable coupling of $\chi$ to the inflaton field $\Phi$ :

$$
V(\chi, \Phi)=\lambda|\chi|^{2}\left(\Phi-\Phi_{1}\right)^{2}
$$

In such a case the "gates" to the flat directions would be open only for a short time when the inflaton field $\Phi$ was close to $\Phi_{1}$. Hence the probability of the penetration to the flat directions is small and $\chi$ could acquire a large expectation value only in a tiny fraction of space. The universe would have a homogeneous background of baryon asymmetry $\eta \sim 6 \cdot 10^{-10}$ generated by the same field $\chi$ which did not penetrate to larger distance through the narrow gate or by another mechanism of baryogenesis, while the regions of high density baryonic matter, $\eta>0$, or antimatter, $\eta<0$, would be rare, but their contribution to the total cosmological mass density might be significant or even dominant. Let us call these bubbles with high baryonic number density B-balls.

Originally the density contrast of B-balls with respect to the average cosmological energy density was very small (isocurvature fluctuations) but after the QCD phase transition such bubbles with large baryonic and/or antibaryonic density would become much heavier than the background medium (of the same volume), so they could form stellar-like astrophysical objects at the very early stage of the cosmological history. As is shown in ref. [12] such antimatter bubbles could survive in the early universe against annihilation with the background of baryonic matter with small asymmetry $\eta \sim 10^{-9}$. Physically it is practically evident because the mean free path of the particles of normal matter in the early universe is very short, so the annihilation could proceed only on the surface of the high-B bubbles, which has quite low efficiency. At later cosmological stages the same reason prevents from strong annihilation again because of a short mean free path inside such bubbles with high baryonic density. These bubbles might form different types of astrophysical objects, from primordial black holes, compact stars, e.g. similar to red giant cores or white dwarfs, or even resemble almost normal stars. Their observational manifestations in the Galaxy, such as e.g. an existence of $\mathrm{MeV}$ photons from the annihilation $e^{+} e^{-} \rightarrow 2 \gamma$ and more energetic $\gamma, e^{-}$, and $e^{+}$from $p \bar{p}$-annihilation, were analyzed in ref. [12], where it was found that no data at the present time are at odds with such a hypothesis.

In the present paper we wish to suggest an alternative way to search for antistars in galaxies through a difference of the polarization of radiation emitted by stars and antistars (it will work for antigalaxies as well). 
Usually it is supposed that in order to determine if the neighboring star is an antistar, the phenomenon of CP-violation should be used. Just after the discovery of CP violation in neutral kaons [13] the following scenario was discussed: the inhabitants of the explored star system were asked, if the shells of their atoms were made from the light charged leptons which were more frequently produced in $K_{L}$ decays $K_{L} \rightarrow \pi^{ \pm} e^{\mp} \nu$. If the answer is "yes" - then we are dealing with an antistar. The problem is that to realize such a scenario we need to establish communication with the inhabitants of another stellar system, which does not seem an easy task.

As it has been noted in [14], if such a scenario can be realized, it assumes communication by radiowaves, so photons emitted on the Earth are detected and analyzed at the stellar system under scrutiny. But in this case $\mathrm{CP}$-violation is not needed: we can send left-handed photons telling, that polarization of charged lepton emitted in neutron $\beta$ decay is mainly the same. This is the way to understand if the investigated system is made from antimatter. But what can we do if the stellar system is not inhabited or we are not able to establish a contact with inhabitants?

So the question we address is how one can distinguish from observations of a given star whether it is a normal star or an antistar? If neutrinos produced in thermonuclear reactions are detectable on the Earth, we will immediately find out, whether it is a star which emits neutrinos, or an antistar which emits antineutrinos. However the flux of neutrinos from stars is too low to be detected: even the observation of neutrinos from the Sun is highly nontrivial: the registered number of events is small. More promising is a supernova explosion. It starts from neutronization reaction $p e^{-} \rightarrow n \nu$, in which neutrinos are emitted. If instead from the first stage of SN explosion antineutrinos are detected on the Earth, it would mean that an antistar exploded [15]. Let us mention that detectors on the Earth waiting for SN explosion in our galaxy are capable to detect neutrinos from neutronization and distinguish them from antineutrinos. (Let us note that spin-flavour conversion of Majorana neutrinos would mimic $\overline{S N}$ explosion [16]. However the spectra of the detected antineutrinos should be different in this case and in the case we consider).

The next question is what one can do if only photons emitted by a star are detected. Usually one would think that the only way to distinguish a star from an antistar is provided by $\mathrm{CP}$ violation. In particular $\mathrm{CP}$ violation leads to a difference in intensity of atomic lines emitted by atoms and antiatoms. Though the energies of emitted photons and the total widths of atomic lines are the same for atoms and antiatoms due to CPT-invarance, the violation of $\mathrm{CP}$ leads to different probabilities of particular transitions in atoms and antiatoms. This way to determine if we are dealing with antistar was suggested in [17]. However since CP violation in atomic transitions is very weak, it would be interesting to find an alternative way to search for antistars.

This way is provided by ordinary weak interaction processes with photon emission. These photons are longitudinally polarized. They could be separated from the overall photon background if they have well defined energy being created e.g. in two body decays. If detected on the Earth such photons would have opposite polarization to that found for the laboratory produced photons, it would mean that they were emitted by an antistar.

Presently beauty meson decays originating from the $b \rightarrow s \gamma$ penguin transition are widely discussed (see [18] and references therein). Since a left-handed $s$-quark is produced in this decay (the probability of a right-handed $s$-quark emission is suppressed as $\left(m_{s} / m_{b}\right)^{2} \sim 10^{-3}$ ) the emitted photon should be left-handed as well. Monoenergetic photons emitted in $B \rightarrow K^{\star} \gamma$ transitions could be used for search of antistars, if there are not two major problems: first, one can hardly imagine production of $B$-mesons in stars since they are too heavy; second, even if $b \bar{b}$ pair is produced, then the beauty quarks would mainly reside in $B$ - and $\bar{B}$-mesons. Hence the photons produced in $B \rightarrow K^{\star} \gamma$ and $\bar{B} \rightarrow K^{\star} \gamma$ decays would have opposite polarizations.

The situation with strange quarks looks more optimistic. First, lower energies are needed to produce strange particles. Second, strange quarks would mainly reside in hyperons. Photons produced in $\Sigma^{+} \rightarrow p \gamma$ decays have large longitudinal polarization, $\alpha=-0.76 \pm 0.08$ [19] and measuring their polarization at the Earth we can see if they were emitted by an antistar (in this case the photon polarization is opposite). Branching of $\Sigma^{+} \rightarrow p \gamma$ decay equals $(1.23 \pm 0.05) \times 10^{-3}$. Stars with considerable amount of strange quarks are discussed in the literature (the so-called strange stars [20] $){ }^{1}$ In outer shell of strange stars considerable amount of $\Sigma$-hyperons should exist and studying the polarization of photons emitted in their decays we can figure out if strange star is in fact an antistrange antistar.

The parity nonconservation in the $\gamma$-transitions of normal nuclei made from protons and neutrons was observed in the Earth experiments [21]. The circular polarization of photons appeared to be rather small: $P_{\gamma}=+(4 \pm 1) \cdot 10^{-5}$ for ${ }^{175} \mathrm{Lu}$ transition with the emission of $395 \mathrm{keV}$ photon; $P_{\gamma}=-(6 \pm 1) \cdot 10^{-6}$ for $482 \mathrm{keV}$ photon emitted in ${ }^{181} \mathrm{Ta}$ transition and $P_{\gamma}=(1.9 \pm 0.3) \cdot 10^{-5}$ for the $1290 \mathrm{keV}$ photon emitted in the $\gamma$-transition of ${ }^{41} \mathrm{~K}$. The observation of the circular polarization of photons with definite energy is ideally suited for the search of antistars.

We are grateful to E. Akhmedov, S. Blinnikov, V. Egorychev, B. Kerbikov and A. Khodjamiryan for useful discussions. The support of the grant of the Russian Federation government 11.G34.31.0047. is acknowledged. V.N. and M.V. are supported by the RFBR under the Grants No. 11-02-00441, 12-02-00193 and by the Russian

\footnotetext{
${ }^{1}$ We are grateful to A. Khodjamiryan for this comment.
} 
Federation Government under Grant NSh-3172.2012.2 as well.

[1] Ya. B. Zeldovich, I.D. Novikov, Structure and Evolution of the Universe, Moscow, Nauka, 1975; A.D. Dolgov, Ya.B. Zeldovich, Rev. Mod. Phys. 53 (1981) 1.

[2] Planck Collaboration, arXiv:1303.5076v1 [astro-ph.CO].

[3] A.D. Sakharov, Pisma Zh. Eksp. Teor. Fiz.5 (1967) 32, JETP Lett. 5 (1967) 24.

[4] R. Duperray, B. Baret, D. Maurin, G. Boudou, A. Barrau, L. Derome, K. Protasov, M. Buenerd, Phys. Rev. D71 (2005) 083013.

[5] K. Abe, H. Fuke, S. Haino S, et al, BESS Collaboration, Phys. Rev. Lett. 108 (2012) 131301.

[6] H. Fuke, T.Malno, K. Abe, et al, BESS Collaboration, Phys. Rev. Lett. 95 (2005) 081101.

[7] G. Steigman, Ann. Rev. Astron. Astrophys. 14 (1976) 339.

[8] G. Steigman, JCAP 0810 (2008) 001.

[9] A.G. Cohen, A. De Rújula, S.L. Glashow, Astrophys. J. 495, 539 (1998); A.G. Cohen, A. De Rújula, astro-ph/9709132.

[10] A. Dolgov, J. Silk, Phys. Rev. D 47 (1993) 4244;

A.D. Dolgov, M. Kawasaki, N. Kevlishvili, Nucl.Phys. B807 (2009) 229.

[11] I. Affleck, M. Dine, Nucl. Phys. B 249 (1985) 361.

[12] C. Bambi, A.D. Dolgov, Nuclear Physics B 784 (2007) 132;

A.D. Dolgov, AIP Conf.Proc. 1116 (2009) 155, arXiv:0901.2100,

[13] J.H. Christenson, J.W. Cronin, V.L. Fitch, R. Turlay, Phys. Rev. Lett. 13 (1964) 138.

[14] L.B. Okun, Leptoni i qvarki, second edition, Moscow, Nauka, 1990.

[15] A.V. Barnes, T.J. Weiler, S. Pakvasa, A.J. 323 (1987) L31.

[16] E. K. Akhmedov, T. Fukuyama, JCAP 0312 (2003) 007.

[17] A.D. Dolgov, I.B. Khriplovich, A.S. Rudenko, JETP Lett. 96 (2012) 421.

[18] The LHCb Collaboration, B.Adeva, et al, arXiv 0912.4179.

[19] J. Beringer et al. RPP, Phys. Rev. D 86 (2012) 1344.

[20] See e.g. G. Pagliara, M. Herzog, F. K. Roepke, arXiv:1304.6884 [astro-ph.HE] and references therein.

[21] V.M. Lobashov, V.A. Nazarenko et al., Pisma v ZhETF 3 (1966) 268;

V.M. Lobashov, V.A. Nazarenko et al., Phys. Lett. B 25 (1967) 104;

V.M. Lobashov, N.A. Lozovoy et al., Phys. Lett B 30 (1969) 39. 